\title{
Research
}

\section{Studying Software Evolution of Large Object-oriented Software Systems using an ETGM Algorithm}

\author{
Segla Kpodjedo ${ }^{a}$, Filippo Ricca ${ }^{b}$, Philippe Galinier $^{a}$, Giuliano Antoniol $^{a}$, and \\ Yann-Gaël Guéhéneuc ${ }^{a}$ \\ ${ }^{a}$ DGIGL / Ptidej Team / SOCCER Lab., École Polytechnique de Montréal, Québec, Canada \\ ${ }^{b}$ DISI, University of Genova, Italy
}

\author{
Studying Software Evolution of Large Object-oriented Software Systems using \\ an ETGM Algorithm \\ Segla Kpodjedo*, Filippo Ricca, Philippe Galinier, Giuliano Antoniol, and Yann-Gael
} Guéhéneuc

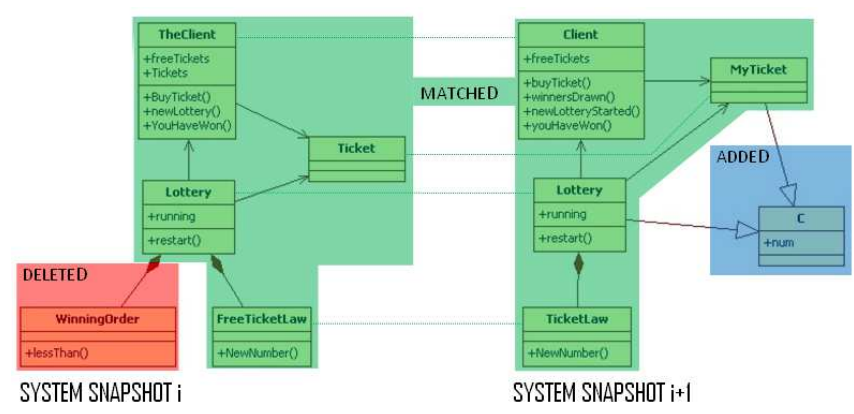

This paper presents a graph matching approach for the study of Object Oriented software systems' evolution. Class diagrams of different releases are modelled as directed graphs and matched using an ErrorTolerant Graph Matching (ETGM) algorithm. classes and thus likely constitute the backbone of the system. An extensive empirical evaluation of our algorithm using small (Rhino), medium (Azureus and ArgoUML), and large systems (Mozilla

and Eclipse) show that our algorithm is fast, scalable and stable.

\section{SUMMARY}

Analyzing and understanding the evolution of large object-oriented software systems is an important but difficult task in which matching algorithms play a fundamental role. An error-tolerant graph matching (ETGM) algorithm can identify evolving classes that maintain a stable structure of relations (associations, inheritances, and aggregations)

\footnotetext{
${ }^{1}$ Correspondence to: Segla Kpodjedo - segla.kpodjedo@polymt.ca
} 
with other classes and thus likely constitute the backbone of the system. Therefore, to study the evolution of class diagrams, we first develop a novel ETGM algorithm, which improves the performance of our previous algorithm. Second, we describe the process of building an oracle to validate the results of our approach to solve the class diagram evolution problem. Third, we report for the new algorithm the impact of its parameters on the F-measure summarizing precision (quantifying the exactness of the solution) and recall (quantifying the completeness of the solution). Finally, with tuned parameters, we carry out and report an extensive empirical evaluation of our algorithm using small (Rhino), medium (Azureus and ArgoUML), and large systems (Mozilla and Eclipse). We thus show that this novel algorithm is scalable, stable and has better time performance than its earlier version.

KEY WORDS: Software evolution, Error-tolerant graph matching algorithms, Oracle. 


\section{INTRODUCTION}

Modern object-oriented (OO) programming languages, such as $\mathrm{C}++$, Java, or C\#, are very popular in academia and industry. Large OO systems exist that have been under continuous development and evolution for many years. When maintaining and evolving such systems, developers must understand the rationale of previous changes and the underlying design decisions. Similarly, managers rely on the history of the design evolution to plan future maintenance and evolution tasks.

However, it is difficult for developers to understand a source code of more than a thousands of lines of code while OO systems can have sizes in the range of millions of lines of code (e.g., Mozilla, OpenOffice, KDE, Gnome, and Eclipse). Moreover, it is difficult and error-prone for developers to compare two sets of lines of code from two different releases of a system. Therefore, finding internal and structural changes and understanding the evolution of classes ${ }^{2}$ in systems is a difficult task for developers, who need tools to help them.

Graph and tree matching algorithms have been proposed in the literature to help developers in identifying structural changes between the designs of subsequent releases of large OO systems $[1,15,23,25,26,27]$. In a typical setting, a design representation, usually the class diagram, is first recovered from the code and then a matching algorithm is applied to gain insight on its evolution. Most of these matching algorithms have been tailored to a specific representation (e.g., XML DOM tree) and only address a specific problem, such as class diagram evolution. Some of them are efficient but it may be difficult to adapt them to a different representation or to tackle a different problem, e.g., the evolution of state or activity diagrams.

In our previous work $[20,21]$, we proposed and applied an error-tolerant graph matching (ETGM) algorithm [19] to analyze and understand the evolution of large OO systems. A graph matching algorithm is an interesting tool for studying software evolution because it makes no assumption on the problem structure but for its graph representation. Moreover, it can be tailored to different problems by modifying its parameters.

For the sake of simplicity and similarly to previous work $[1,21,26]$, we assume that the essential design information of systems is captured by their class diagrams. This assumption does not reduce the generality of our algorithm, which can be applied on any representation, provided that they can be converted into directed graphs.

Our novel algorithm takes as input two graphs representing the UML class diagrams of two releases of a system. These diagrams may be crafted by hand or reverse-engineered from two releases of the source code and-or binaries. It then produces a matching of the two graphs. The costs assigned to matching distortions, i.e., possible differences between matched elements, inherent in any such algorithm, are tuned to fit the class-diagram matching problem. The result is a list of matched classes, matched relations, and insertions and removals of classes and relations between the two releases.

In this paper, we extend our previous work [21] with four contributions. First, we present a new and faster ETGM algorithm. Second, we detail the process of building an oracle to

${ }^{2}$ In the rest of this paper, we talk about "classes" for the sake of simplicity with no loss of generality if interfaces and structures exist. 
validate the results of our algorithm. This oracle, built for three systems (Rhino, ArgoUML, and Azureus), is the first of its size reported in the literature. Third, we provide a study of the behavior of our ETGM algorithm via the F-measure [12], when changing its parameter values. Finally, we study the scalability and stability ${ }^{3}$ of the new algorithm using five systems (Rhino, Azureus, ArgoUML, the Mozilla Web browser, and Eclipse).

This paper is organized as follows. Section 2 details the class diagram evolution problem and presents the theory behind our ETGM algorithm. Section 3 describes the context and research questions of our empirical evaluation of the algorithm. It also introduces a process to build an oracle to study such algorithm and apply it on three systems: Rhino, ArgoUML, and Azureus. Section 4 reports and discusses the results of the evaluation. Section 5 treats qualitative analysis and threats to the validity of our evaluation. Finally, Section 6 presents related work and Section 7 concludes and outlines future work.

\section{BACKGROUND}

During maintenance tasks, developers must understand the evolution of the design of a system. As suggested in [26], novice developers rely on the evolution history of a design to take decisions and get inspiration when fixing new problems. Managers also use evolution history to find evolution patterns symptomatic of problems to prevent and avoid in the future. Therefore, a tool able to analyze the evolution of a system, reporting (un)stable classes and relations can help both developers and managers.

In the following, we present background information on the evolution of class diagrams, and on the theory and application of our ETGM algorithm to this problem.

\subsection{Class Diagram Evolution Problem}

Let us consider two versions $D_{1}$ and $D_{2}$ of the class diagram of a system, observed at two different instants in time, and let $C l\left(D_{1}\right)$ and $C l\left(D_{2}\right)$ denote the set of classes in $D_{1}$ and $D_{2}$. A matching between $C l\left(D_{1}\right)$ and $C l\left(D_{2}\right)$ is a relation between the two sets, i.e., a subset of $C l\left(D_{1}\right) \times C l\left(D_{2}\right)$. Figure 1 displays an example of $D_{1}$ and $D_{2}$. An optimal matching between $C l\left(D_{1}\right)$ and $C l\left(D_{2}\right)$ representing the actual evolution of the system would be the following solution, i.e., the class WinningOrder was deleted; a new class $C$ was created; classes TheClient, Ticket, FreeTicketLaw were renamed into Client, MyTicket, TicketLaw.

Thus, given two class diagrams, the class diagram evolution (CDE) problem consists in finding, among the large set of all possible matchings, a solution that is the true matching between $C l\left(D_{1}\right)$ and $C l\left(D_{2}\right)$. The quality of a solution, for example produced by an automatic technique, can be evaluated with classic information retrieval measures, such as precision, recall, and F-measure [12].

${ }^{3}$ Our algorithm is stochastic, thus its results can vary from one run to the next. Its stability must be studied to assess how large can be the variations.

Copyright (C) 2000 John Wiley \& Sons, Ltd.

J. Softw. Maint. Evol.: Res. Pract. 2000; 00:1-1 

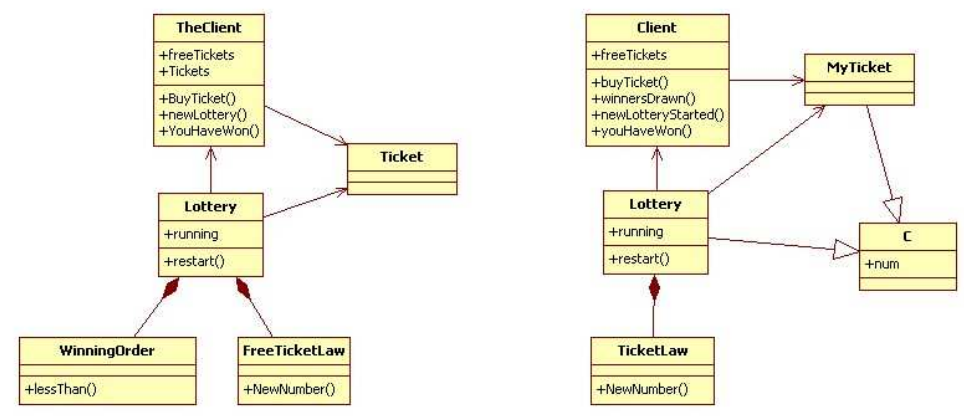

Figure 1. Example of class diagrams to be matched

In the following, we do not take merge and split operations of classes into account, i.e., matching between two classes in different versions are always one-to-one. Therefore, we represent a matching by a partial function $m: C l\left(D_{1}\right) \rightarrow C l\left(D_{2}\right)$ such that one class of $D_{1}$ is matched to at most one class of $D_{2}$.

\subsection{Available Information}

Given two class diagrams, a developer or an algorithm can use different kinds of information to find the true matching, in particular the internal similarity between classes, i.e., names of the classes and information from their attributes and methods, and structural information, i.e., the relations among classes.

\subsubsection{Names of the Classes}

In our previous work $[1,20,21]$, we observed empirically that class names are stable, i.e., they do not change dramatically over time. Therefore, if two classes in two different releases have a same name, they are likely to be the same class and constitute a valid match.

Consequently, a naive algorithm could use class names and match any two classes with a same name. Yet, two classes can share the same name, be coded in the same file, but have important differences in their implementation, their public interface, and their role in the system. Classes can be renamed or moved from one version to another and thus be missed by a naive algorithm (e.g., when developers translate class names from a language to another).

Different measures of string distance could then be used to identify (some) renamed classes but also with limitations because many classes can share similar names. Moreover, to the best of our knowledge, there is no string distance yet able to give a measure based on the lexical meaning of strings. For instance, from ArgoUML 0.16.1 to ArgoUML 0.17.5, the class GoGenElementToDerived was renamed to GoGeneralizableElementToSpecialized. Such a renaming, while obvious for a developer, yields a high string distance. 
Therefore, we use names of classes in conjunction with information extracted from their methods, attributes, and relations.

\subsubsection{Interface Information}

Interface information refers to the sets of attributes and methods in the two classes publicly available. We use an extended definition of interface in that we consider that all methods and all attributes are part of the interface regardless of their visibilities.

We observed empirically that when classes are renamed, their interfaces remain similar: their attributes and methods do not change dramatically [1,20,21]. Consequently, if two classes present a high level of similarity in their interface, they are likely to be the same class.

However, interface similarity between classes does not always provide precise and accurate information. The interface of some classes does not provide any valuable information because it is too small or because the fields and methods are generic, e.g., the two fields $\mathrm{x}$ and y may be found in many classes dealing with geometry.

\subsubsection{Structural Information}

Structural information includes the relations among classes present in two class diagrams. This information is useful and reliable in our experience [1, 20, 21]. For example, in Figure 1, we were able to find a matching without resorting to other internal information by only considering association, aggregation, and inheritance relations. The triplet (Lottery,TheClient,Ticket) in the first diagram generates a micro-architecture easily identifiable in the second diagram.

\subsection{Solving the CDE using an ETGM Algorithm}

To solve the CDE problem with the available information, we propose an algorithm based on internal and structural information.

We model the class diagrams of two releases as labeled graphs, with nodes being classes and edges representing the relations among classes. Labels on edges specify the type of the relation: association, aggregation, or inheritance; labels on nodes encode class properties: class name, methods and attributes information.

We then translate the CDE problem into an optimization problem: the differences between the two class diagrams stem from edit operations with assigned costs, accounting for both internal and structural differences. Given some cost parameter values, we propose to solve the optimization problem, using an error-tolerant graph matching algorithm to find a solution with minimal cost.

\subsubsection{Error-tolerant Graph Matching}

A graph with labels from two finite alphabets of symbols $\Sigma_{V}$ (vertices' labels) and $\Sigma_{E}$ (edges' labels) is defined as a triple $\left(V, L_{V}, L_{E}\right)$ where $V$ is the finite set of elements, called nodes or vertices; $L_{V}: V \rightarrow \Sigma_{V}$ and $L_{E}: V \times V \rightarrow \Sigma_{E}$ are the node and edge labeling functions. 
Let $G_{1}=\left(V_{1}, L_{V 1}, L_{E 1}\right)$ and $G_{2}=\left(V_{2}, L_{V 2}, L_{E 2}\right)$ be two graphs. We define a matching as a bijective function $m: \hat{V}_{1} \rightarrow \hat{V}_{2}$ where $\hat{V}_{1} \subseteq V_{1}, \hat{V}_{2} \subseteq V_{2}$. We say $x_{1} \in \hat{V}_{1}$ is matched to node $x_{2} \in \hat{V}_{2}$ if $m\left(x_{1}\right)=x_{2}$. Moreover, any edge $\left(x_{1}, y_{1}\right) \in \hat{V}_{1} \times \hat{V}_{1}$ is said to be matched to the edge $\left(x_{2}, y_{2}\right) \in \hat{V}_{2} \times \hat{V}_{2}$ if $m\left(x_{1}\right)=x_{2}$ and $m\left(y_{1}\right)=y_{2}$.

All unmatched nodes $\left(V_{1}-\hat{V}_{1}\right)$ and unmatched edges $\left(V_{1} \times V_{1}-\hat{V}_{1} \times \hat{V}_{1}\right)$ from $G_{1}$ are said to be deleted while all unmatched nodes $\left(V_{2}-\hat{V}_{2}\right)$ and unmatched edges $\left(V_{2} \times V_{2}-\hat{V}_{2} \times \hat{V}_{2}\right)$ from $G_{2}$ are said to be inserted.

Matches, deletions, insertions of nodes and edges are the basic edit operations applied to transform $G_{1}$ into $G_{2}$. Costs are assigned to these operations, depending on the desired matching. The cost of a matching $m$ between two graphs is the sum of the costs of all edit operations induced by $m$.

We are not interested in computing an isomorphism between the two graphs because, unlike isomorphism, evolution is precisely about changes. Therefore, we use an approximate - hence error tolerant- graph matching algorithm, which finds a matching with the lowest possible cost. Further details on ETGM algorithms can be found in [7].

\subsubsection{From Class Diagrams to Graphs}

Two class diagrams $D_{1}$ and $D_{2}$ are represented by two labeled graphs $G_{1}$ and $G_{2}$. To model a class diagram as a graph, we proceed as follows:

- Each class $c$ in the class diagram is represented by a node $x_{c}$ in the graph: the class name (path/package included) becomes the node name while attributes and methods' signatures are encoded as properties of the node using textual representations. An attribute is encoded by collapsing its type, visibility, and name in a string; a method signature is a string obtained by collapsing its name, the type(s) of its parameter(s), and its return type. Each class is characterized by a triple $(l, A, M)$, with $l$ the class name, $A$ the set of attributes, and $M$ the set of methods.

- Relations are assigned letters: $a$ for association, $b$ for composition/aggregation, and $c$ for inheritance. Given two classes $c_{1}$ and $c_{2}$, the edge label $w=l\left(x_{c_{1}}, x_{c_{2}}\right)$ is the string obtained by concatenating the letters that correspond to the relation between $c_{1}$ and $c_{2}$. For instance $w=a b b$ means that, from $c_{1}$ to $c_{2}$, there are: one relation of type $a$ (association) and two relations of type $b$ (aggregation). If there is no relation between the two classes, we have $l\left(x_{c_{1}}, x_{c_{2}}\right)=\lambda$ (where $\lambda$ denotes the empty string).

\subsubsection{Cost Parameter Values}

A node match occurs when a class $c_{1}$ in $G_{1}$ is matched to a class $c_{2}$ in $G_{2}$. A cost must be paid for this match if the information of $c_{1}$ and $c_{2}$ are different. This cost depends on the dissimilarity between class names and the internal information. Information from class name, attributes, and methods are combined using three weights: $l_{w}$ (for class name), $m_{w}$ (for methods), and $a_{w}$ (for attributes), which are three real values in $[0,1]$, such that $l_{w}+m_{w}+a_{w}=1$. The dissimilarity value is normalized between zero (when $c_{1}$ and $c_{2}$ are identical) and one (when the two classes have nothing in common). Thus, a node match between two classes $c_{1}$ and $c_{2}$ 
$\operatorname{costs} c_{n m} \times$ dissimilarity $\left(c_{1}, c_{2}\right)$, where $c_{n m}$ is the maximal cost for a node match and the dissimilarity between two classes $c_{1}$ and $c_{2}$ is:

$$
\begin{aligned}
\operatorname{dissimilarity}\left(c_{1}, c_{2}\right) & =l_{w} \times\left(\frac{\text { Levenshtein }\left(l_{1}, l_{2}\right)}{\max \left(\text { length }\left(l_{1}\right), \text { length }\left(l_{2}\right)\right)}\right) \\
& +m_{w} \times\left(1-\frac{2 \times\left|M_{1} \cap M_{2}\right|}{\left|M_{1}\right|+\left|M_{2}\right|}\right) \\
& +a_{w} \times\left(1-\frac{2 \times\left|A_{1} \cap A_{2}\right|}{\left|A_{1}\right|+\left|A_{2}\right|}\right)
\end{aligned}
$$

where Levenshtein is the Levenshtein distance that is a computationally-efficient way to evaluate the string edit distance [9]; $M_{1}$ (respectively, $M_{2}$ ) represents the set of methods of $c_{1}$ (respectively, $c_{2}$ ); $A_{1}$ (respectively, $A_{2}$ ) represents the set of attributes of $c_{1}$ (respectively, $c_{2}$ ). The intersection of the sets of attributes $A_{1} \cap A_{2}$ (respectively methods $M_{1} \cap M_{2}$ ) contains the attributes (respectively methods) that did not go through a single change (renaming, type change, and so on), i.e., their textual representations are perfectly similar.

An edge match occurs when relations in the first diagram are matched to their counterparts in the second diagram: every couple of matched nodes $\left(c_{1}, s_{1}\right)$ from the first diagram is matched to $\left(m\left(c_{1}\right), m\left(s_{1}\right)\right)$. Given $l\left(c_{1}, s_{1}\right)=w_{1}$ and $l\left(m\left(c_{1}\right), m\left(s_{1}\right)\right)=w_{2}$, we call $\phi\left(w_{1}, w_{2}\right)$ the cost of the edge match and we distinguish four cases depending on the labels of the edges:

1. $\phi(w, w)=0$ when $w_{1}=w_{2}=w$;

2. $\phi\left(w_{1}, \lambda\right)=$ length $\left(w_{1}\right) \times c_{\text {emd }}$ when $w_{1} \neq \lambda$ and $w_{2}=\lambda$;

3. $\phi\left(\lambda, w_{2}\right)=\operatorname{length}\left(w_{2}\right) \times c_{e m i}$ when $w_{1}=\lambda$ and $w_{2} \neq \lambda$;

4. $\phi\left(w_{1}, w_{2}\right)=\left(\right.$ length $\left(w_{1}\right)-$ length $\left.(w)\right) \times c_{\text {emd }}+\left(\right.$ length $\left.\left(w_{2}\right)-\operatorname{length}(w)\right) \times c_{\text {emi }}$ when $w_{1} \neq \lambda, w_{2} \neq \lambda, w_{1} \neq w_{2}$ and where $w$ the common part between $w_{1}$ and $w_{2}$.

When the two edge labels are identical (Case 1), no cost is required. Cases 2 and 3 correspond to a structural error. A cost $c_{e m d}$ is paid for each relation removal (i.e., relations present in the first diagram but missing in the second) and a $c_{e m i}$ for each relation addition (i.e., relations present in the second diagram but missing in the first). Case 4 corresponds to a label error and it can be viewed as a two-phase operation: (1) remove relations (from the first diagram) missing in the second diagram and (2) add the relations (of the second diagram) missing in the first diagram: Case 2 plus Case 3. This case is consistent with the reality of code evolution and spares us the need to specifically assign a cost to label errors.

Two other cost parameter values, $c_{n d}$ and $c_{n i}$, are assigned to class deletions and insertions. Unmatched relations (adjacent to deleted classes) of the first diagram are deleted and generate, each, a cost $c_{\text {eud }}$ while unmatched relations (adjacent to inserted classes) of the second diagram are inserted and generate, each, a cost $c_{\text {eui }}$.

Consequently, each potential matching $m$ is assigned a cost $f(m)$. This cost is the sum:

$$
f(m)=f_{\text {node_err }}(m)+f_{\text {node_unmatched }}(m)+f_{\text {edge_err }}(m)+f_{\text {edge_unmatched }}(m)
$$

where $f_{\text {node_err }}(m)$ corresponds to penalties related to node label dissimilarity; $f_{\text {node_unmatched }}(m)$ corresponds to node deletions and insertions; $f_{\text {edge_err }}(m)$ corresponds to 
Table I. ETGM cost parameters

\begin{tabular}{|l|l|}
\hline Parameters & Description \\
\hline$c_{n m}$ & Maximum cost of a match between two classes \\
\hline$l_{w}$ & $l_{w}, a_{w}, m_{w}$ are real numbers between 0 and 1 such as $l_{w}+a_{w}+m_{w}=1 ;$ \\
$a_{w}, m_{w}$ & they weight respectively information about class name, attributes and methods \\
\hline$c_{n d}$ & cost of deleting a class present in $V_{1}$ but missing from $V_{2}$ \\
\hline$c_{n i}$ & cost of adding a class present in $V_{2}$ but missing from $V_{1}$ \\
\hline$c_{e m d}$ & cost of deleting a relation linking two classes of $V_{1}$, both present in $V_{2}$ \\
\hline$c_{e m i}$ & cost of adding a relation linking two classes of $V_{1}$, both present in $V_{2}$ \\
\hline$c_{e u d}$ & deleting a relation linking two classes of $V_{1}$, of which at least one is missing from $V_{2}$ \\
\hline$c_{e u i}$ & adding a relation linking two classes in $V_{2}$, of which at least one is missing from $V_{1}$ \\
\hline
\end{tabular}

penalties related to edge label discrepancies; and finally, $f_{\text {edge_unmatched }}$ corresponds to edge deletions or insertions. These terms are computed as follows:

- $f_{\text {node_err }}(m)=c_{n m} \times \Sigma_{x_{c} \in \hat{V}_{1}}$ dissimilarity $(c, m(c))$;

- $f_{\text {node_unmatched }}(m)=c_{n d} \times\left|V_{1}-\hat{V}_{1}\right|+c_{n i} \times\left|V_{2}-\hat{V}_{2}\right|$;

- $f_{\text {edge_err }}(m)=\Sigma_{(x, y) \in \hat{V}_{1} \times \hat{V}_{1}} \phi(l(x, y), l(m(x), m(y)))$;

- $f_{\text {edge_unmatched }}(m)=c_{\text {eud }} \times\left|\left\{(x, y) \in V_{1} \times V_{1}-\hat{V}_{1} \times \hat{V}_{1}: l(x, y) \neq \lambda\right\}\right|+c_{\text {eui }} \times \mid\{(x, y) \in$ $\left.V_{2} \times V_{2}-\hat{V}_{2} \times \hat{V}_{2}: l(x, y) \neq \lambda\right\} \mid$.

where $V_{1}$ (respectively, $V_{2}$ ) is the set of classes from the first diagram (respectively, the second diagram), $\hat{V}_{1}$ is the matched subset of $V_{1}$ and $\hat{V}_{2}$ is the matched subset of $V_{2}$.

Thus, ten cost parameters, $l_{w}, a_{w}, m_{w}, c_{n m}, c_{n d}, c_{n i}, c_{e m d}, c_{e m i}, c_{e u d}$, and $c_{e u i}$ - see Table I) - are used in the cost model of the ETGM algorithm when solving the CDE problem.

\subsubsection{Tuning the ETGM Cost Model}

By trial-and-error experiments on multiple systems, we observed that a multiplication of the cost parameters by a constant do not affect the results. Indeed, cost parameters do not influence the optimal matching as absolute values but as ratios. These ratios lead to a more or less greater tolerance from the ETGM algorithm to errors and-or more or less greater importance to different kinds of information.

In essence, the developer can decide what is important for her in the result set: if she favors matching on the basis of the structure or the linguistic information (class name, methods' signatures and attributes), if renaming of classes should be admissible, and so on.

We define five aggregate parameters - see Table II - that specify the kind of matching to be expected: dropWeightNode and dropWeightEdge to calibrate error tolerance, edgeWeight and labelWeight to calibrate the importance of different sources of information, and asymmetry to take into account the direction of the matching.

2.3.4.1. Calibrating Error Tolerance. When two nodes $n_{1}$ from $g_{1}$ and $n_{2}$ from $g_{2}$ are matched, a cost expressing their dissimilarity $\left(c_{n m} \times \operatorname{dissimilarity}\left(n_{1}, n_{2}\right)\right)$ must be paid; 
Table II. ETGM Aggregate parameters

\begin{tabular}{|c|c|c|}
\hline \multirow{5}{*}{$\begin{array}{l}\text { Parameters } \\
\text { dropWeightNode } \\
(d w n)\end{array}$} & \multicolumn{2}{|c|}{ Description } \\
\hline & Role & considering only internal changes, drop or match? \\
\hline & Formula & $d w n=\frac{c_{n d}+c_{n i}}{c_{n m}}$ \\
\hline & Range & $\begin{array}{l}\text { Min }=0: \text { zero-tolerance on internal changes; } \\
\text { Max }=1 \text { : no penalty for class dissimilarity }\end{array}$ \\
\hline & Void if & edgeWeight $\rightarrow \infty$ \\
\hline \multirow[t]{4}{*}{ dropWeightEdge } & Role & considering only relational changes, drop or match? \\
\hline & Formula & $d w e=\frac{c_{e u d}+c_{e u i}}{c_{e m d}+c_{e m i}}$ \\
\hline & Range & $\begin{array}{l}\text { Min }=0 \text { : zero-tolerance on relational changes; } \\
\operatorname{Max}=1 \text { : no penalty for relational changes }\end{array}$ \\
\hline & Void if & edgeWeight $\rightarrow 0$ \\
\hline \multirow{3}{*}{$\begin{array}{l}\text { edgeWeight } \\
(e w)\end{array}$} & Role & structural information over class internal information? \\
\hline & Formula & $e w=\frac{c_{e m d}+c_{e m i}}{c_{n m}}$ \\
\hline & Range & $\begin{array}{l}\text { Min }=0 \text { : structural information is not considered; } \\
\text { Max } \rightarrow \infty \text { : only structural information is considered }\end{array}$ \\
\hline \multirow{3}{*}{$\begin{array}{l}\text { asymmetry } \\
(\text { asy) }\end{array}$} & Role & additions over deletions? \\
\hline & Formula & $a s y=\frac{c_{n i}}{c_{n d}}=\frac{c_{e u i}}{c_{e u d}}=\frac{c_{e m i}}{c_{e m d}}$ \\
\hline & Range & $\begin{array}{l}\text { Min }=0 \text { : additions are penalty-free; } \\
\operatorname{Max} \rightarrow \infty \text { : deletions are penalty-free }\end{array}$ \\
\hline \multirow{4}{*}{$\begin{array}{l}\text { labelWeight } \\
(l w)\end{array}$} & Role & class name over attributes and methods? \\
\hline & Formula & $l_{w}=1-\left(a_{w}+m_{w}\right)$ \\
\hline & Range & $\begin{array}{l}\text { Min=0: zero-tolerance on internal changes; } \\
\text { Max=1: no penalty for class dissimilarity }\end{array}$ \\
\hline & Void if & edgeWeight $\rightarrow \infty$ \\
\hline
\end{tabular}

otherwise, a cost for deleting $n_{1}$ and inserting $n_{2}\left(c_{n d}+c_{n i}\right)$ is paid. The dropWeightNode parameter calibrates the level of tolerance to dissimilarity between two classes. It defines a threshold of dissimilarity beyond which the cost paid in case of a match is higher to the cost paid when the classes are not matched.

Similarly to the $d r o p W e i g h t N o d e$, we define $d r o p W e i g h t E d g e$ to compare two alternatives: matching two edges or excluding them from the solution. Perfect edge matches always yield to a zero cost but when we have differences between matched edges, we must know how much more we must pay.

The higher the values of dropWeightNode or dropWeightEdge, the higher the tolerance to errors in the solution. When those parameters have a value of 1 or higher, our ETGM algorithm becomes "error-friendly", i.e., the worst matches are equal or better than any set of deletion and insertion. Values of 0 lead to an "error-free" configuration, which allows only perfect matches. 
2.3.4.2. Calibrating the Importance of Different Sources of Information. The edgeWeight parameter indicates the importance of structural information over internal information in the solution. The higher this parameter value, the higher the importance of structural information in the solution. A 0 value means that the structural information is dismissed while a very high value (near $\infty$ ) means that internal information is irrelevant. The labelWeight parameter allows ignoring either interface information (labelWeight $=1$ ) or class name information $($ labelWeight $=0)$, or finely tuning their contribution in the matching.

2.3.4.3. Taking into Account the Direction of Matching. We could assume that matching $G_{1}$ with $G_{2}$ or $G_{2}$ with $G_{1}$ makes use of a same cost model. However, because software system evolves in the direction of time, we define the asymmetry parameter to take into account the direction of a matching. Asymmetry means that edit operations in one direction may cost more than the same operations in the other direction. An asymmetry value of (i) 0 means that additions do not count, (ii) 1 that additions have the same weight as removals, (iii) $\infty$ that removals do not count.

When setting the asymmetry parameter to a value different from 1, this parameter should also be used in the computation of the dissimilarity between two classes. Given two classes, we must then replace Equation 1 by Equation 2:

$$
\begin{aligned}
\operatorname{dissimilarity}\left(c_{1}, c_{2}\right) & =l_{w} \times\left(\frac{\text { Levenshtein }\left(l_{1}, l_{2}\right)}{\max \left(\text { length }\left(l_{1}\right), \text { length }\left(l_{2}\right)\right)}\right) \\
& +m_{w} \times\left(1-\frac{\left|M_{1} \cap M_{2}\right|+\text { asymmetry } \times\left|M_{1} \cap M_{2}\right|}{\left|M_{1}\right|+\text { asymmetry } \times\left|M_{2}\right|}\right) \\
& +a_{w} \times\left(1-\frac{\left|M_{1} \cap M_{2}\right|+\text { asymmetry } \times\left|M_{1} \cap M_{2}\right|}{\left|A_{1}\right|+\text { asymmetry } \times\left|A_{2}\right|}\right)
\end{aligned}
$$

\subsection{Implementing the ETGM Algorithm using a Tabu Search}

The ETGM problem is known to be NP-hard [24]. Therefore, the only algorithms that can find optimal solutions have an exponential worst-case complexity, i.e., they require prohibitive computation times even for medium-size graphs. Therefore, we resort to heuristics to obtain near optimal solutions in acceptable computation times.

\subsubsection{Tabu Search-based Implementation}

We use a tabu search algorithm to implement our ETGM algorithm. Starting from an initial solution, the tabu search algorithm moves iteratively from the current solution to a neighboring one. At each iteration, the algorithm chooses the best neighbor, i.e., the solution $S$ with the best cost according to our cost model, while avoiding returning toward solutions recently visited by using a short-term tabu list [13].

The cost of a solution $S$ is the sum of the costs of the edit operations induced by $S$. A move applied to the current solution consists in (1) inserting into $S$ a new match between two nodes (respecting the one-to-one constraint) or (2) removing a match from $S$. 
The tabu list forbids recently-inserted node matches to leave the solution for a given number of iterations (randomly chosen between 5 and 10) and recently-removed node matches to reenter the solution for a given number of iterations (randomly ${ }^{4}$ chosen between 10 and 20).

The stop criterion is a number of iterations without improvements of the best solution found.

\subsubsection{Initialization of the Tabu Search-based ETGM Algorithm}

In our experiments, we observed that the tabu implementation does not perform well when initialized with an empty solution. It wanders in the search space for a very large number of iterations looking for better solutions. The same problem happens when the algorithm is initialized using a random solution. However, it performs well when initialized with a solution that already contains a significant number of true matches.

We propose two different initialization procedures:

- a procedure SI_Init() that uses structural information to build the initial solution;

- a procedure Names_Init() that uses the names of the classes to build the initial solution.

The first procedure uses structural information. It uses local similarity to build an initial solution. For any node $x$ and for any $l \in \Sigma_{E}$, we denote by $f_{x}(l,+)$ and $f_{x}(l,-)$ the numbers of incoming and outgoing edges adjacent to $x$ and whose labels is $l$. We measure the local similarity of all pairs of nodes $x_{1}$ and $x_{2}$ using the maximal number of common edges between $x_{1}$ and $x_{2}$. Then, we sort the pairs according to their similarity, in decreasing order. Each pair is then considered in turn. If the current pair is compatible with all the pairs already in the solution and if it does not degrade its quality, then it is inserted in the solution.

The second procedure matches two classes whenever they have the same name. It requires the names of the classes to provide reliable information, which may not always be the case (e.g., when a large-scale class renaming took place).

Overall, our ETGM algorithm is able to match two class diagrams as explained above and produces pairs of classes as well as added and deleted classes. For example, if it is applied to the class diagram in Figure 1, with the cost parameters identified in the sensitivity analysis (see Section 4), then it will report the pairs shown in the left part of Table III while the right part of the table shows the information that the algorithm gathered about the differences between the two class diagrams: the attribute, methods, and relations changes. Currently, however, our algorithm does not detect if a method or attribute is moved to a different class not matched with the original one. We will consider this case in future work.

\footnotetext{
${ }^{4}$ Random selection of a number in a given interval is a well-known technique aimed at further preventing cycling during a local search. Difference between tabu lengths of leaving and re-entering matches account for the fact that, due to the one-to-one matching scheme, matches present in the solution forbid many more moves. Chosen values result from preliminary tests.
} 
Table III. Matches and reported changes

\begin{tabular}{|c|c|c|c|c|c|}
\hline \multirow{2}{*}{ Class in $V_{1}$} & \multirow{2}{*}{ Class in $V_{2}$} & \multicolumn{2}{|c|}{ Internal } & \multicolumn{2}{|c|}{ Structural } \\
\hline & & Changes & Costs & Changes & Costs \\
\hline TheClient & Client & $\begin{array}{l}\text { Renaming } \\
-1 \text { attribute } \\
+1 \text { method }\end{array}$ & 36 & - & 0 \\
\hline FreeTicketLaw & TicketLaw & Renaming & 18 & - & 0 \\
\hline Ticket & MyTicket & Renaming & 14 & +1 inheritance & 8 \\
\hline Lottery & Lottery & - & 0 & $\begin{array}{l}-1 \text { composition } \\
+1 \text { inheritance }\end{array}$ & 11 \\
\hline WinningOrder & - & Deletion & 33 & - & 0 \\
\hline- & $C$ & Insertion & 17 & - & 0 \\
\hline
\end{tabular}

\section{EMPIRICAL EVALUATION}

In this section, we introduce our empirical study, including the process used to build the oracle needed to validate the results. The main goal of our empirical evaluation is to investigate the applicability of our ETGM algorithm to solve the CDE problem. We are also interested in (1) studying the effect of changing the cost parameters and determining (2) the scalability of the algorithm and (3) the stability of its results across several runs. The quality focus is sensitivity, scalability, and stability of our ETGM algorithm. We want to study how our ETGM algorithm reacts to small changes of its parameters, if it scales up to large systems, and if different runs produce consistent results. The perspective is both of researchers, who often use class diagrams to study software evolution, and of developers, who want to quickly find some insights on the evolution of large OO systems. The context of the evaluation are five open-source systems, detailed in the next subsection.

\subsection{Objects}

We perform our evaluation using five well-known systems of various sizes: Rhino, ArgoUML, Azureus, the Mozilla Web browser, and Eclipse. Rhino ${ }^{5}$, the smallest system, is a JavaScript/ECMAScript interpreter and compiler that implements the ECMAScript international standard, ECMA-262 v3 [10]. We use the 15 releases between 1.4R3 to 1.7R1, downloaded from its Web site. Release 1.5R6 consists of 32,134 source lines of Java code (excluding comments and blank lines), 138 classes, 1,339 attributes, and 1,870 methods.

Azureus $^{6}$ (recently renamed Vuze) is a medium-size, Java, BitTorrent client. Azureus supports the BitTorrent protocol to exchange and distribute data over the Internet. We only use the releases "2" of Azureus: the 18 releases from 2.0.3.2 to 2.5. Release 2.5 contains 2,628 classes with about 9,700 attributes, and 18,000 methods, for a size of 450 KLOCs.

${ }^{5}$ http://www.mozilla.org/rhino/

${ }^{6}$ http://azureus.sourceforge.net/ 
$\mathrm{ArgoUML}^{7}$ is a medium-size, Java-based, UML development tool. It was first released in 1998 and has been actively developed and evolved since. It supports most of the standard UML diagrams and allows the use of OCL constraints. It can also export data in a variety of formats, including XMI, C++, C\#, Java, and PHP source code. We analyzed 9 releases of ArgoUML from 0.10 to 0.2. Release 0.2 contains 1,493 classes with 4,128 attributes, 9,659 methods, for a size of 300 KLOCs.

Mozilla $^{8}$ is a large open-source suite implementing a Web browser and other tools, such as a mailer. It was ported on almost all software and hardware platforms. It is developed mostly in $\mathrm{C}++$, with $\mathrm{C}$ code accounting for only a small fraction of the system. Differently from our previous work, we focus on the 24 releases of the Web browser, from 1.0 to 1.7.12. We select these 24 releases because they were used in another previous work [3]. The Mozilla Web browser release 1.7.12 includes 6,892 classes, 20,018 attributes, and 48,048 methods, for a size of 3.5 MLOCs (2.5 MLOCs C++ code).

Eclipse $^{9}$ is a large, open-source, integrated development environment. It is a platform used both in the open-source community and in industry, for example as a base for the WebSphere family of development environments. Eclipse is mostly written in Java, with $\mathrm{C} / \mathrm{C}++$ code used mainly for the widget toolkit. $\mathrm{C}++$ code is not considered in this study. We use releases 1.0, 2.0.0, 2.1.1, 2.1.2, and 2.1.3. Release 2.1.3 contains 7,648 classes with 50,636 attributes, 89,341 methods, in more than 8,000 files for about 2.4 MLOCs.

We recover the class diagrams of the five systems using the Ptidej tool suite [16] suite and its PADL meta-model. PADL is a language-independent meta-model to describe the static part and part of the behavior of object-oriented systems in a similar fashion to UML class diagrams. It includes a Java parser and a dedicated graph exporter. We recover the class diagrams of the Mozilla Web browser through the AOL intermediate format [2]. AOL is a language to textually describe UML-like class diagrams. Parsers exist for various programming languages, including $\mathrm{C}$ and $\mathrm{C}++$. All artifacts (list of releases and graph representations of the class diagrams) in this study are available from the SOCCER laboratory Web server, under the Software Evolution Repository (SER) site, accessible at http://web. soccerlab.polymtl.ca/SER/.

\subsection{Research Questions}

This study refines and extends the research questions presented in our previous work [21] in several ways. We now use five systems instead of one to study the scalability of our ETGM algorithm. We study for the first time the sensitivity of the results to the parameters and compare the results of our novel algorithm with those of our previous ETGM algorithm. Consequently, we examine the following research questions:

- RQ1 - ETGM Sensitivity: What is the effect on the solutions of changing the ETGM five aggregate cost parameters?

\footnotetext{
${ }^{7}$ http://argouml.tigris.org/

${ }^{8}$ http://www.mozilla.org/

${ }^{9}$ http://www.eclipse.org/
} 
- RQ2 - ETGM Scalability: How is the performance of our ETGM algorithm impacted when the size of the class diagram varies from small (e.g., Rhino) to large (e.g., Eclipse).

- RQ3 - ETGM Stability: Are different runs of our ETGM algorithm on the same inputs producing consistent and stable solutions?

RQ1 aims at providing insights on the effect of cost parameters on the accuracy of the obtained solutions to determine which parameters are more important and if there exist a set of parameter values producing solutions very close to an oracle. This question is preliminary to the two other ones. RQ2 targets scalability; we select five systems of different sizes to investigate the impact of the size of the class diagrams on matching time. RQ3 aims at providing a measure of the stability of the returned solutions; our algorithm, like most meta-heuristics, is stochastic and there is no guarantee of obtaining identical solutions for different runs. ${ }^{10}$.

\subsection{Analysis Method}

We perform different analyses to answer the three research questions:

- RQ1: We run ten times our ETGM algorithm on each pair of subsequent releases of Rhino, ArgoUML, and Azureus for various values of cost parameters and compare the solutions with a built oracle.

- RQ2: We apply our ETGM algorithm to pairs of releases of the five systems and record and compare the computation times.

- RQ3: We run ten times our ETGM algorithm on the same pairs of releases and compare solutions to verify if different runs produce consistent solutions, i.e., containing the same class pairs.

Sensitivity must be quantified in a precise and non-ambiguous way with respect to the oracle. Different metrics are needed for RQ1 and RQ3. Answering RQ1 requires quantifying, with respect to the oracle, the number of correctly matched classes as well as of unmatched classes. To answer RQ1, we consequently use the measure of precision and recall [12]. Furthermore, we use an aggregate measure, the $F$-Measure [12], which is a standard combination of precision and recall, defined as their harmonic mean:

$$
F=\frac{2 \times \text { precision } \times \text { recall }}{\text { precision }+ \text { recall }}
$$

Some of the five parameters may be more relevant than the others to improve the quality of the solutions. Therefore, we study the sensitivity of the ETGM algorithm into a 5-dimension space. One problem of representation arises as more than three dimensions are difficult to model, handle, and visualize.

Consequently, first, for each system, we assign for each parameter a set of values. Then, we build all possible combinations of the parameters and run the ETGM algorithm ten times

\footnotetext{
${ }^{10}$ Note however that our algorithm can be easily rendered deterministic by fixing the random seed used for the runs.
} 
for each pair of subsequent releases of the system. After each run, we compute the precision and recall of the obtained solutions with respect to the oracle. We then plot the F-measure of the average of each parameter combinations to assess the influence of the chosen parameters on the ETGM algorithm. The maximum values of the F-measure correspond to a balanced compromise between precision and recall; values before the peak favor precision while values after promote recall.

To answer RQ2, we run two distinct experiments. Indeed, the time required to find a solution to the CDE problem depends on two factors: the size of the two input graphs and their differences (i.e., their delta). In the first experiment, we compare each pair of subsequent releases of the five systems; while in the second, we use the oldest release as a reference point and we match it with all subsequent releases. In each experiment, we run the ETGM algorithm ten times for each system and each pair of releases and record the resulting computation times.

To answer RQ3, we perform an analysis similar to that in our previous work [21]: we quantify similarity between the $n$-computed solutions using the Jaccard index. The Jaccard index is a statistic tool used for comparing the similarity of sample sets. It provides a percentage of similarity defined as the size of the intersection divided by the size of the union of the sample sets multiplied by 100. In essence, we would like that different ETGM runs contain the same class pairs. If, in one run, a given class pair is not contained while it is contained in the other $n-1$ runs, then we count one instability.

\subsection{Building an Oracle for the ETGM Algorithm}

An oracle is needed to study our ETGM algorithm and validate its matchings for the CDE problem. Such an oracle must provide, for a set of systems and a set of versions the true matchings between different pairs of systems and versions.

Manually building the mapping between two releases is a time consuming task even for systems of the size of Rhino (the smallest among the analyzed systems). Indeed, for each pair of Rhino releases, there are about 10,000 candidate matches. Producing an oracle for medium systems of the size of Azureus or ArgoUML may require to inspect millions of matches and an exhaustive manual verification is no longer feasible. Clearly, a manual mapping is impossible for larger systems such as Mozilla and Eclipse.

However, in most cases, software evolution is a continuous and smooth process where drastic restructuring rarely changes the system structure completely. Between two subsequent releases, renaming does not affect all the classes and most of the code is not rewritten. As a consequence, for any given two releases, most of possible class pairs between class diagrams are false positives, i.e., matches that should be discarded. Unfortunately, only the developers implementing a change hold the knowledge of their intent and can say if the change was meant to evolve the class or merely save its interface.

Let us consider the case where the class interface and the class name remain the same but the semantics of methods become different. Based on the Bunge ontology $[5,6]$, we believe that those classes are really different because their behavior is different. Consistently with previous work [14], it is our opinion that there is a blurred boundary between refactoring or evolving a class and the two operations of deleting a class and adding a new one. The boundary is further blurred by the possible drift of class semantics across several releases; at the end of the 
$n^{\text {th }}$ release, a class may be totally different from the class in the initial release but each small increment did not drastically change its previous behavior and interface. Overall, only a fine grain analysis of the CVS/SVN repository change log may provide hints on the developers' intent and the class evolution path.

However, most of the time, a class interface, relations with other classes, and relative position in the file system are sufficient to decide if it should be matched to another in the subsequent version. For example, if between two subsequent releases, the class name and position in the system were not changed and the attribute types and class signature were not modified, then there is no reason to believe that the class was completely reimplemented. If the class size substantially changes, an inspection of the CVS/SVN repository may help to decide between a match and a false positive.

The semi-automated oracle building process that we implemented is based on the above considerations while attempting to reduce the required manual verification. It consists of three main phases: two automatic phases followed by a manual verification on a reduced subset of matching candidates. First, we compare each class of one version with each class of the other. When comparing two classes, we define a similarity of attributes and methods. Attribute similarity uses attribute names and types; method similarity combines methods name and signatures (formal parameter and returned types). We assume that if the two classes: (1) have the same name, (2) are in the same position of the system (i.e., package location), and (3) have methods and attributes with similarity values above a given threshold, then they are really the same class, i.e., one is the evolution of the other. To compute attributes and methods similarity, we use Equation 1. To build the oracle, we set the similarity threshold at 50\%. This threshold and the similarity computation process are more constraining than that of UMLDiff $[27,26]$, which assumes that a class is unchanged if its name and its package are the same.

In the second automatic phase, pairs discarded in the first phase are reanalyzed to identify possible match candidates to be manually inspected in the last phase. We consider two cases. First, given a class pair in which none of the classes was part of a match computed in the previous phase, if they have at least three common elements, and:

- the class names have a similarity above the threshold;

- or their fields declarations have a similarity above the threshold;

- or their method declarations have a similarity above the threshold;

then the pair is passed forward to manual verification. Second, if one of the two classes was also already tagged as part of a match or was considered as potentially matchable, then we apply the same rules to pass or not the pair to the manual verification, with a warning that extra attention must be paid as the classes are possibly evolving from/to more than one class.

All pairs passed on to the manual verification are inspected by experts. In this paper, the authors played the role of experts. Although they are not knowledgeable of the specific systems, each of them has at least five years of experience in developing OO systems with Java and $\mathrm{C}++$. While the generation of pairs to be checked is automatic, the manual verification of those pairs required about two days of work for Rhino, ArgoUML, and Azureus. 


\section{EVALUATION RESULTS}

We now present the results of our empirical evaluation.

\subsection{RQ1 - ETGM Sensitivity}

We compared the solutions produced by our ETGM algorithm with the solutions available in the oracle under different initial parameters. We studied the five aggregate parameters presented in Section 2.3.4: dropWeightNode, dropWeightEdge, edgeWeight, asymmetry, and labelWeight. These parameters are ratios which relevant ranges are $[0,1]$.

There is theoretically an infinite number of combinations of parameters. Consequently, we reduced the analysis space by sampling uniformly with step 0.25 the interval $[0,1]^{5}$ for Rhino, ArgoUML, and Azureus.

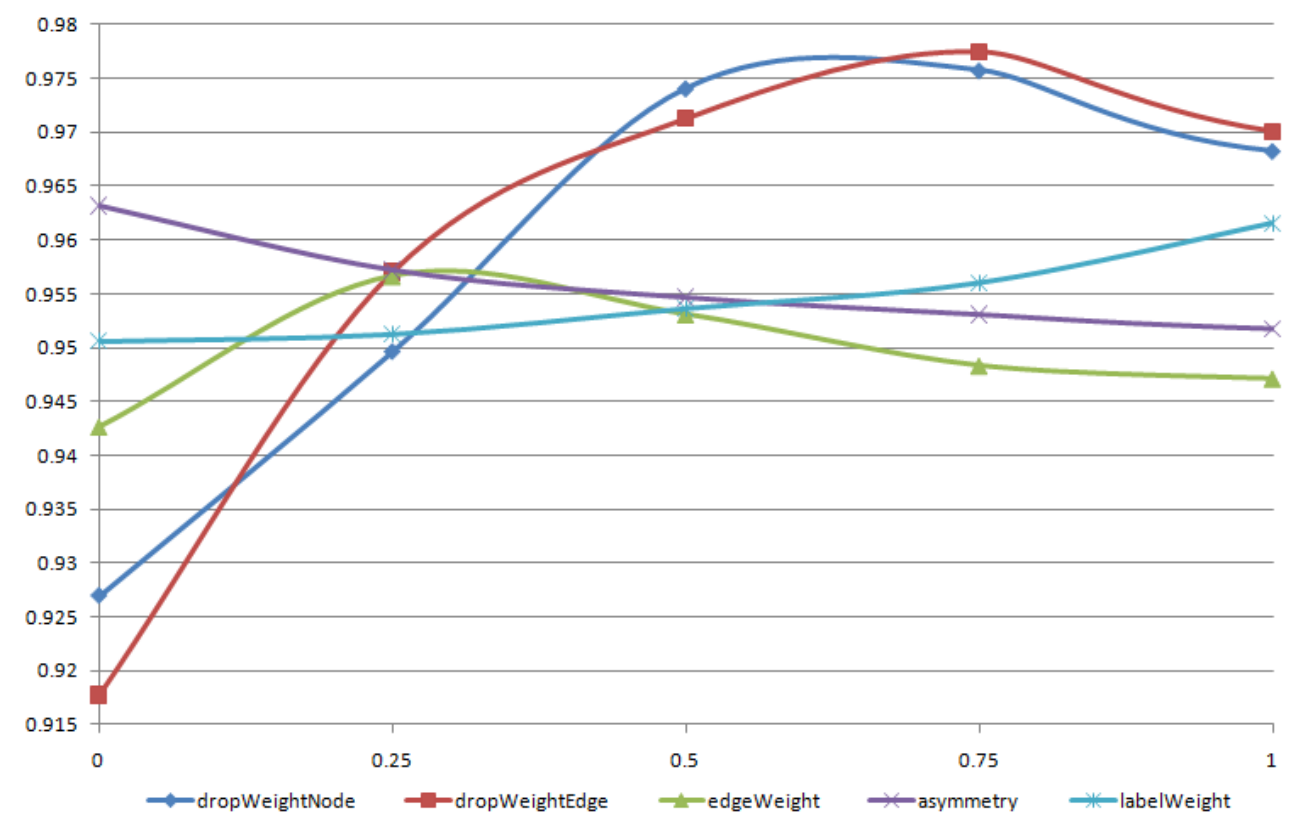

Figure 2. Rhino sensitivity analysis: F-measure depending on the aggregate parameters

Figures 2, 3, and 4 report the means of $\mathrm{F}$-measures for the different aggregate parameters on the three systems. The F-measures for ArgoUML and Azureus are translated rightwise wrt. that of Rhino. We explain this fact by the particularities of Rhino evolution. In Rhino, few class splitting occurred and, thus, our ETGM algorithm is able to obtain one-to-one matches while in ArgoUML and Azureus, class splitting occurred more frequently and our algorithm returns only one match. 


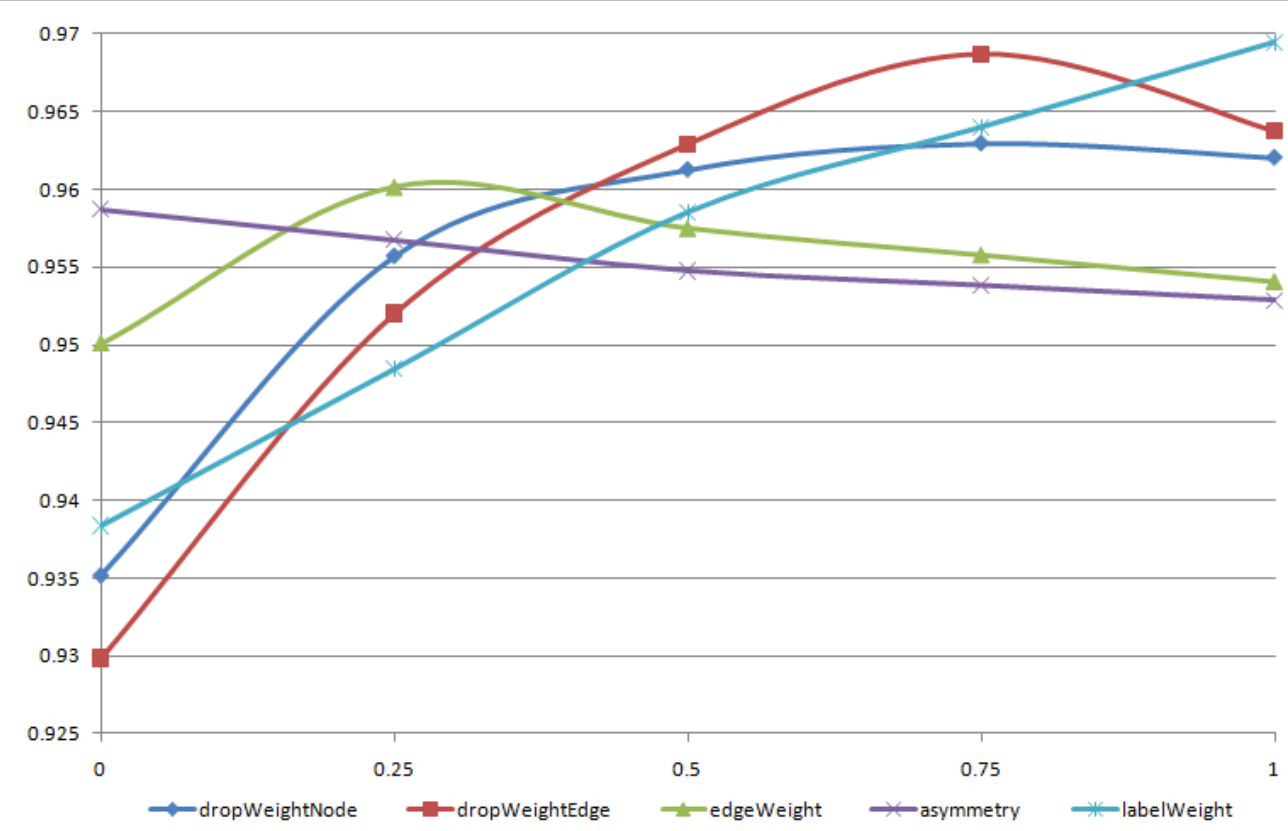

Figure 3. ArgoUML sensitivity analysis: F-measure depending on the aggregate parameters

For all three systems, the figures show that the parameters dropWeightNode and dropWeightEdge are more relevant because they lead to large variations in the F-measure. The other three parameters only marginally impact the F-measure. For example, Figure 3 shows that edgeWeight causes a variation of the F-measure between 0.95 and 0.96 , i.e., it impacts only the second decimal.

We thus conclude that there exists a region of the parameters space where a compromise between precision and recall is obtained and where our ETGM algorithm returns solutions closely similar to those in the oracle. A fine-tuning of the parameters allows us to select the values favoring precision and recall for the three systems: values of 0.5 for dropWeightNode, dropWeightEdge, and asymmetry, of 0.1 for edgeWeight, and of 0.6 for labelWeight yield a good compromise for the F-measure value, with an average close to 0.97. Such an F-measure value on our data set ensures a precision and recall close or higher to 0.95 .

We set a medium error tolerance to class dissimilarity and structural errors. With an edgeW eight of 0.1, structural information is not expected to impact dramatically the matching process but only serves as a small complement to internal information. With asymmetry $=0.5$, we set a preference in the direction of the matching, meaning additions are twice less expensive than removals in the matching process. We also gave a slight advantage to class name, labelWeight $=0.6$, over class methods and attributes, as the name of a class is often an important clue during its evolution.

We use these values in the following subsections. 


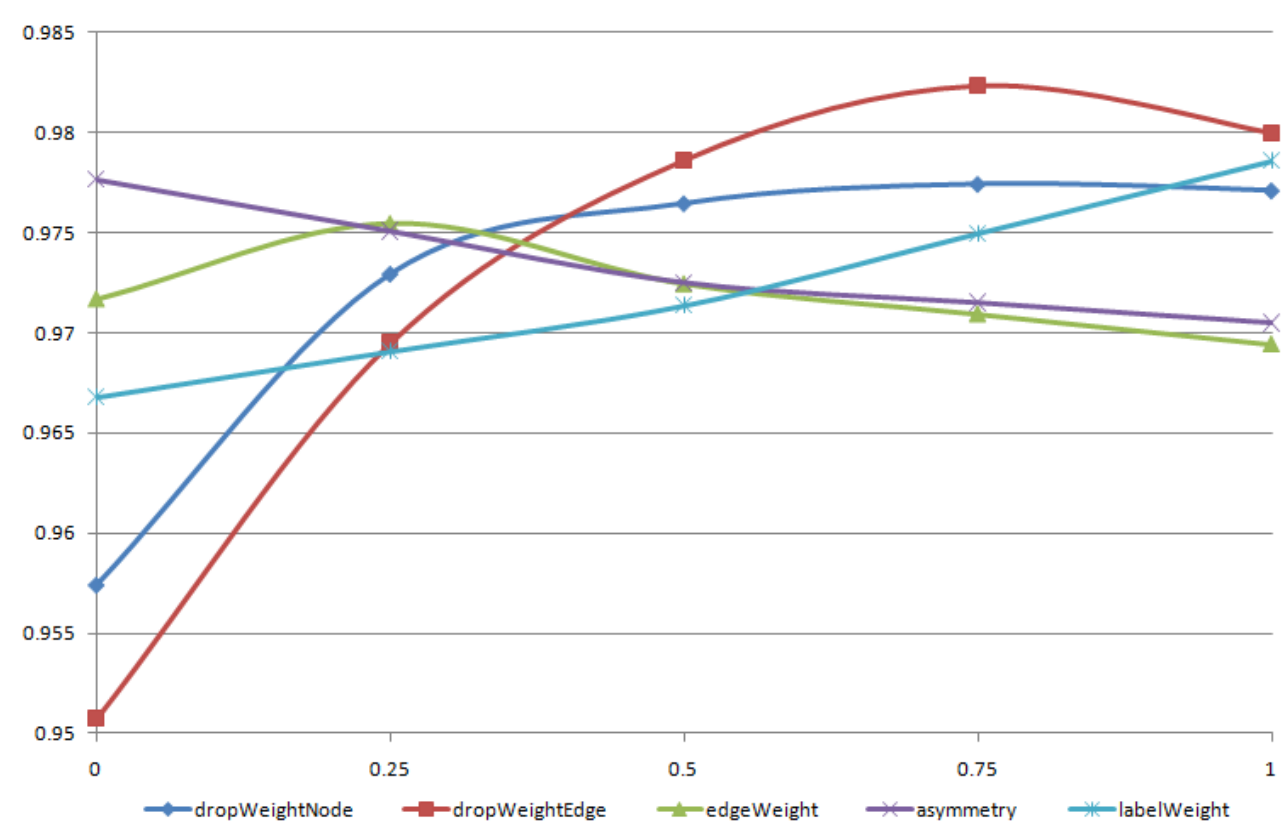

Figure 4. Azureus sensitivity analysis: F-measure depending on the aggregate parameters

Table IV. Matching time (seconds) between subsequent releases.

\begin{tabular}{|l|l|l|l|}
\hline \multirow{2}{*}{ Systems } & \multicolumn{3}{|l|}{ Running Times (seconds) } \\
& Mean & Std. Dev. & Max. \\
\hline Rhino & 0.06 & 0.02 & 0.12 \\
ArgoUML & 2.96 & 2.49 & 7.38 \\
Azureus & 1.45 & 1.18 & 4.05 \\
Mozilla & 18.76 & 2.58 & 28.48 \\
Eclipse & 158.2 & 177.34 & 477.0 \\
\hline
\end{tabular}

Table V. Matching time (seconds) against the oldest releases.

\begin{tabular}{|l|l|l|l|}
\hline \multirow{2}{*}{ System } & \multicolumn{3}{|c|}{ Running Times (seconds) } \\
& Mean & Std. Dev. & Max. \\
\hline Rhino & 0.23 & 0.11 & 0.43 \\
ArgoUML & 20.0 & 11.0 & 45.5 \\
Azureus & 6.15 & 4.63 & 16.28 \\
Mozilla & 85.42 & 25.16 & 115.18 \\
Eclipse & 763.0 & 162.18 & 943.0 \\
\hline
\end{tabular}

\subsection{RQ2 - ETGM Scalability}

The impact of the sizes of the graphs of the two versions of a system on the time required to find a solution to the CDE problem depends on two factors. The size of the two input graphs is important as well as their differences. Therefore, we run the two experiments described in Section 3.3. We performed all computations on a dual Opteron server, with 16GB of RAM, running RedHat Advanced Server. Process size for the experiment was limited to 8GB. For all runs, except for Eclipse, memory usage never exceeded 4GB. 
Table VI. Stability for subsequent releases - Jaccard index of matched

\begin{tabular}{|l|l|l|l|}
\multicolumn{4}{c}{ pairs. } \\
\hline Application & Mean & Std. Dev & Min \\
\hline Rhino & 100 & 0 & 100 \\
ArgoUML & 98.81 & 1.32 & 96.5 \\
Azureus & 99.33 & 2.24 & 90.76 \\
Mozilla & 99.83 & 0.23 & 99.11 \\
Eclipse & 99.65 & 1.72 & 96.41 \\
\hline
\end{tabular}

Table VIII. Stability for subsequent releases - Jaccard index of inserted

\begin{tabular}{|l|l|l|l|}
\hline \multicolumn{5}{|c}{ classes. } \\
\hline Application & Mean & Std. Dev & Min \\
\hline Rhino & 100 & 0 & 100 \\
ArgoUML & 98.66 & 3.4 & 90.27 \\
Azureus & 99.65 & 0.87 & 96.8 \\
Mozilla & 96.34 & 7.37 & 75.00 \\
Eclipse & 99.28 & 0.9 & 98.13 \\
\hline
\end{tabular}

Table VII. Stability for non-subsequent releases - Jaccard index of matched

\begin{tabular}{|l|l|l|l|}
\multicolumn{4}{c}{ pairs. } \\
\hline Application & Mean & Std. Dev & Min \\
\hline Rhino & 98.82 & 2.30 & 93.94 \\
ArgoUML & 88.80 & 9.39 & 72.96 \\
Azureus & 81.63 & 8.85 & 62.13 \\
Mozilla & 96.35 & 1.42 & 95.12 \\
Eclipse & 93.58 & 3.33 & 88.76 \\
\hline
\end{tabular}

Table IX. Stability for non-subsequent releases - Jaccard index of inserted

\begin{tabular}{|l|l|l|l|}
\multicolumn{5}{c}{ classes. } \\
\hline Application & Mean & Std. Dev & Min \\
\hline Rhino & 99.75 & 0.47 & 98.68 \\
ArgoUML & 97.63 & 1.64 & 95.98 \\
Azureus & 98.95 & 0.56 & 97.47 \\
Mozilla & 96.67 & 0.62 & 95.77 \\
Eclipse & 97.97 & 0.27 & 97.57 \\
\hline
\end{tabular}

Table IV and V report the means, standard deviations, and maximum running times for each system in the two experiments. Computation times are in general below the minute but for Eclipse, with a maximum of 8 minutes. Therefore, we conclude that our ETGM algorithm is practical and could be run as part of a normal development process in the industry.

Computation times on Eclipse are substantially higher than those of other systems due to the matching of Eclipse releases 1.0 and 2.0. Indeed, these two releases are quite different. Release 1.0 contains 6,188 classes while release 2.0 has 9,027 classes. A large part of the classes in release 2.0 did not exist in the 1.0 and thus cannot be matched. The ETGM algorithm spends time finding the best possible matchings for about 3,000 classes without match.

Table $\mathrm{V}$ reports the impact of the sizes of the graphs on the computation times when matching each subsequent release with the oldest release of each system. As time passes, the new releases are more and more different from the earliest one and computation times increase. Confirming our previous analysis, the most time-consuming computations are for Eclipse, last row of the table. The maximum time, about 15 minutes, occurs when matching Eclipse releases 1.0 and 2.1.3; the graph representation of release 1.0 contains 6,188 classes while the 2.1.3 class diagram contains 10,870 classes.

\subsection{RQ3 - ETGM Stability}

We reuse the results of the matchings performed to answer RQ2 to quantify the stability of our ETGM algorithm using the Jaccard index. Tables VI, VIII, and X show the Jaccard indexes for matched, inserted, and deleted classes for subsequent releases. Each ETGM run produces 
Table X. Stability for subsequent releases - Jaccard index of deleted classes.

\begin{tabular}{|l|l|l|l|}
\hline Application & Mean & Std. Dev & Min \\
\hline Rhino & 100 & 0 & 100 \\
ArgoUML & 95.94 & 5.2 & 85.18 \\
Azureus & 99.24 & 2.23 & 91.66 \\
Mozilla & 94.39 & 11.17 & 61.11 \\
Eclipse & 98.44 & 1.25 & 97.56 \\
\hline
\end{tabular}

Table XI. Stability for non-subsequent releases - Jaccard index of deleted

\begin{tabular}{|l|l|l|l|}
\multicolumn{5}{c}{ classes. } \\
\hline Application & Mean & Std. Dev & Min \\
\hline Rhino & 99.06 & 2.32 & 92.86 \\
ArgoUML & 93.72 & 4.13 & 85.19 \\
Azureus & 90.65 & 4.31 & 81.48 \\
Mozilla & 89.35 & 3.83 & 85.45 \\
Eclipse & 97.87 & 1.33 & 96.10 \\
\hline
\end{tabular}

a data point, a pair of matched releases. After ten runs for each release pair, the Jaccard index was computed. In the tables, we report the average, standard deviation, and minimum Jaccard indexes for each system.

Data supports our claim that the ETGM algorithm stability is high. Sometimes, for class pairs with matching cost close to the cost of deleting and adding the classes, the algorithm randomly chooses one of the two possibilities, which impacts, albeit marginally, the stability.

Table VII, IX, and XI report the Jaccard indices quantifying the stability of our algorithm for matched, inserted, and deleted classes, for non-subsequent releases. As the releases are more distant in time, not only do computation times increase but also different matchings are found. Minor differences in inserted or deleted classes cause ripple effects on matching costs, which lead to the Jaccard indices going as low as $60 \%$. In general, the minimum Jaccard indices were acceptable, except in few cases where the low values may jeopardize the algorithm applicability. However, it is important to highlight that in computing the stability, we used the most severe constraint: if a class match is not present in one run but is present in the other nine, or if the matching cost is different in one run, stability decreases, as explained in the following section.

\section{QUALITATIVE ANALYSIS}

We now further discuss the results of our study. We also briefly describe threats to its validity and compare our ETGM algorithm and its results with tool, UMLDiff [26, 27].

\subsection{Generality}

The five analyzed systems exhibit different evolution histories. Rhino is relatively stable; its size increased smoothly from its release 1.4R3 (100 classes) to release 1.7R1 (305 classes). Similarly, ArgoUML size went from 898 classes, in release 0.1, to 1,493 classes in release 0.2. The size of the Mozilla Web browser remained almost constant over the years; its 1.0 release contained 6,866 classes while the last analyzed release 1.7.12 contains 6,892 classes. Mozilla largest release in our data set is 1.7 .2 with 6,920 classes. Eclipse almost doubled the number of its classes from 6,188 in release 1.0 to 10,870 in release 2.1.3. The most dynamic system is Azureus: its first release, 0.3.2, contained 214 classes, while the release immediately following 
(i.e., 0.4) almost doubled, with 384 classes. Azureus reached 980 classes in its third release, 1.0 , and almost doubled again in release 3.0 with 1,959 classes and a final size of 2,628 classes in release 5.0. The history dynamics impact the accuracy and the stability of our algorithm. To some extent, the interpretation of results reported for RQ2 and RQ3 are intermixed.

\subsection{RQ2 - ETGM Scalability}

The ETGM algorithm is implemented in $\mathrm{C}++$, compiled with $\mathrm{g}++$, and run on a Linux Opteron 64bit with 16GB of RAM, as in the experiments reported in our previous work. In [21], the average computation time on the Mozilla suite for a matching of two 15-day-apart snapshots was of about 380 seconds, while the overall matching process took about 15 hours.

The Mozilla Web browser is between 1,000 and 2,000 classes smaller than the Mozilla suite, which has a size smaller or comparable to Eclipse, i.e., between 8,000 and 10,000 classes. Apart from matching Eclipse release 1.0 to 2.0, which takes about 470 seconds, other subsequent Eclipse matchings take between 45 and 110 seconds, as shown in Table IV. Similarly, for all matchings of two subsequent releases of the Mozilla Web browser, running the ETGM algorithm takes less than 25 seconds. Furthermore, when the new ETGM algorithm is run on the snapshots of the Mozilla suite [21], a single run takes an average of 32 seconds (worst performance in ten runs was 42 seconds). These 32 (42) seconds can be directly compared with the 382 seconds reported in our previous work [21]. Thus, we conclude that our novel algorithm runs substantially faster than our previous algorithm.

In agreement with related work [26], we believe that the relative distance of two UML diagrams should be low to favor matching and that it affects stability. On a 2-week distance between class diagrams, the previous ETGM algorithm required about 380 seconds while our novel ETGM algorithm runs faster but for Eclipse releases 1.0 and 2.0. This last result can be explained considering that the delta between the releases 1.0 and 2.0 is of about 3,000 classes $(6,188$ versus 9,029). As shown in Table V, the more different the releases, the longer, on average, the computation times.

\subsection{RQ3 - ETGM Stability}

The stability of our previous ETGM algorithm was high when using 15-day apart snapshots [21]. On the same data set, our new ETGM algorithm has an average stability computed over ten runs of $99.96 \%$, a standard deviation of 0.11 , and a minimum value of $99.19 \%$. This value must be compared to the previous value of $100 \%$ and, thus, it indicates a slight decrease in stability. This negligible decrease is compensated by the reduced computation time, which is a magnitude faster for the novel algorithm.

The objects of our empirical evaluation are of different nature, we thus investigate the results reported in Tables VI, VIII, and X and in Tables VII, IX, and XI. When computing stability, we use ten runs of our algorithm. Therefore, even small discrepancies between the ten computed solutions may lead to large deviations in percentage. Therefore, in the following, we discuss the lowest stability percentages and explain them in terms of absolute numbers.

The lower value of stability for inserted classes, in Table VIII, is obtained for Mozilla, with $75 \%$, when matching releases 1.7 .8 and 1.7.10. Only five classes out of about 6,800 are 


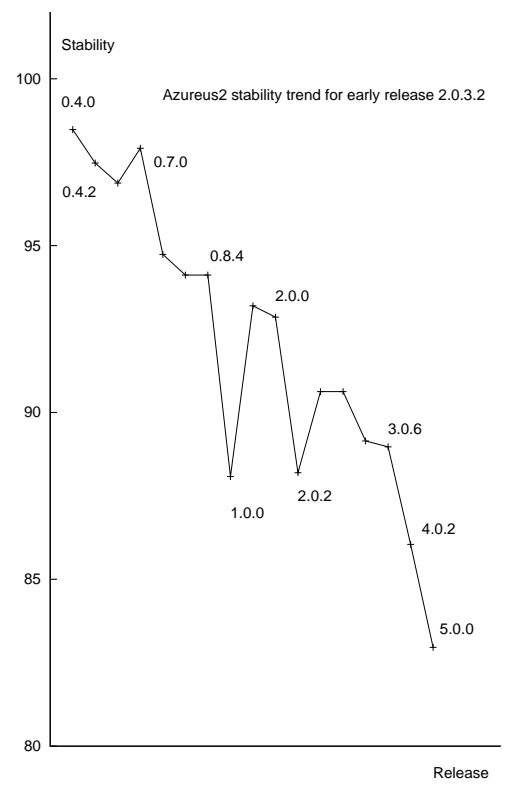

Figure 5. Azureus stability trends of matched classes. Release 0.3.2 compared to following releases and matching cost not considered

fluctuating between matchings due to the non-deterministic nature of our algorithm. Out of the ten runs, the class data is matched with itself nine times while the class nsWindowCreator only two times. We discovered that class nsWindowCreator has only a constructor and that its constructor signature was modified; the cost of deleting and adding nsWindowCreator or matching it with a modified constructor are very close. Not matching nsWindowCreator (thus deleting and adding it) increases the cost of the matching by only 5 with respect to a total cost of 4,000. A similar reasoning can be done for the four other classes. The match of release 1.7.8 with 1.7 .10 is also the one having the lower stability of deleted classes, 61\%. Again, in absolute numbers, only six classes fluctuate between the ten computed solutions.

The Mozilla deletion/insertion dynamics can also be observed on ArgoUML, in particular when matching releases 0.14 and 0.15.6. There are 136 distinct inserted classes out of which 122 are always inserted. Of the 14 remaining classes, two are inserted nine times, NotationProvided and EmptyNode. The classes Generator2 and NotationProvider2 are inserted eight times. Although the two releases contain 1,288 and 1,266 classes, the delta in number of classes hides a heavy refactoring impacting our ETGM algorithm. For example, all classes with names beginning by MenuResourceBounds, UMLCognitiveResourceBoundle, History, and Column were removed in version 0.15.6.

The lowest stability value for matched classes is reached with Azureus, when matching releases 0.3.2 and 0.4.0. This is not a surprise as the delta in number of classes is high, with 
Table XII. ETGM stability for nonsubsequent releases - Jaccard index of matched classes without considering the matching cost.

\begin{tabular}{|l|l|l|l|}
\hline Application & Mean & Std. Dev & Min \\
\hline Rhino & 99.56 & 0.9 & 96.92 \\
ArgoUML & 92.28 & 5.55 & 85.28 \\
Azureus & 92.24 & 4.45 & 82.96 \\
Mozilla & 96.58 & 1.36 & 95.49 \\
Eclipse & 95.55 & 0.82 & 94.76 \\
\hline
\end{tabular}

141 classes added in release 0.4.0. A deeper analysis reveals that if the cost of the matching is disregarded, only three classes fluctuate: DiskManagerFileImpl, TRTrackerServerPeer, and TRTrackerServerPeerImpl, which yields $98.5 \%$ stability.

The analysis of data reported in Tables VII, IX, and XI for non-subsequent versions, further confirmed the findings outlined in Tables VI, VIII, and X. For example, Azureus has $62.13 \%$ stability as lower bound when matching releases 0.3 .2 with 2.0. The same three classes as in our previous experiment, DiskManagerFileImpl, TRTrackerServerPeer, and TRTrackerServerPeerImpl, are involved in fluctuations. Indeed, there are 206 matchings out of which 187 are always present in the ten computed solutions and, if cost is disregarded, the three classes fluctuating between matching and deletion and insertion are again DiskManagerFileImpl, TRTrackerServerPeer, and TRTrackerServerPeerImpl.

To further gain insight on stability, we computed the Jaccard indexes between the ten computations, disregarding the matching costs, for non-subsequent releases of the five systems. Results reported in Table XII show that the stability of the algorithm is much higher than that reported in Table VII and that the decrease of stability when compared to our previous work is reduced. The new ETGM algorithm is much faster with only a negligible loss in stability. We also investigated the effect of the distance in time for Azureus, the system with the greatest history dynamics, growing from 214 classes to 2,628 classes. Figure 5 reports average stability over ten computations when matching its first release 0.3 .2 with all subsequent releases. The marked decreasing stability corresponds to the increase in number of classes: the more different the releases, the more variability in stability.

\subsection{Threats to Validity}

External validity threats concern the generalization of the results of our evaluation. We applied our algorithm on five systems of different sizes, languages and domains. We believe that our results support evidence that an ETGM algorithm can be effectively applied to the CDE problem of large OO systems and that results may be of interest to developers and managers. Still, other systems may provide different results in terms of parameter sensitivity, stability, and computation times. Overall, we believe that, with appropriate tuning of its parameters, our algorithm can be successfully applied even to other software artifacts, including call graphs or UML activity diagrams. 
Construct validity threats concern the relationship between the theory and the observation. Such threats can be due to errors introduced by measurement instruments. Our technique to reverse-engineer class diagrams may be imprecise at times and there is a need to compare our diagrams with others'. Nevertheless, all class diagrams were produced using the same technique to ensure consistency across computations. We cannot exclude the possibility that by using a different reverse-engineering technique, our ETGM algorithm may produce slightly different results. We fixed parameters using an in-depth analysis of their relations to computed solutions, using an oracle. We provided evidence that different parameters produce different matchings and, thus, may impact accuracy. We can only claim that on three of five systems, we found evidence that a set of parameter values leads to solutions with very high precision and recall (i.e., greater than 0.95). However, precision and recall are also tied to the oracle and we cannot claim that it is devoid of errors.

Internal validity refers to the influence of independent variables on dependent variables and the existence of confounding factors. We believe that given the problem addressed in this paper, internal validity threat is not relevant.

Reliability validity concerns the possibility of replicating the study and obtaining the same results. All studied systems are open source and publicly available; the details of the algorithm are presented in this paper and more information can be found in previous papers (e.g., [21]). Finally, all data used in this study can be downloaded from the SER repository.

\subsection{Comparison with the State-of-the-art}

A subset of threats to validity are somehow related to the nature of our algorithm. Other approaches such as UMLDiff [26, 27] may produce comparable results with different pros and cons. Similarly to our algorithm, UMLDiff takes as input two class diagrams, corresponding to the designs extracted from two versions of a system, and produces a list of changes, which reports the differences between the two diagram versions. UMLDiff traverses the containment spanning trees of the two design diagrams implicit in the two graphs and identifies the corresponding classes.

We tackle the same problem but adopt a different and more general approach as our algorithm works directly on graphs. Furthermore, by changing its parameters, our ETGM algorithm can be tuned to match other types of artifacts (e.g., call graphs or UML state diagrams). Moreover, tree matching algorithms may suffer from the order in which sub-trees are loaded and linked into the overall tree structure, making the tree matching less efficient. ETGM matching algorithms do not have such a problem.

In [26], the authors suggested that the relative distance of the two UML diagrams should be low to favor matching and this suggestion is reflected in our results as well. Our study showed that the ETGM algorithm produces deterministic results for similar UML class diagrams with low variability, even for diagrams distant in time.

Our algorithm, in its current version, is less stable than UMLDiff, which is a deterministic algorithm and, thus, will always produce the same results. We could achieve the same stability by forcing our algorithm to always choose the same move when facing a choice between exaequo moves. However, we prefer to use a stochastic algorithm because it gives a better idea of the search space and the range of results that can be obtained. 
In terms of scalability, the case study on which UMLDiff was applied [26, 27] was JFreeChart, whose versions always contain less than 800 classes. The reported computation time is on average about 10 minutes and up to 50 minutes. Our algorithm, though not usable on-line, scales up to 7,500 classes with a computation time of about 2 minutes. It should be noticed though that while our computations were done on a dual Opteron server, with 16GB of RAM, UMLDiff experiments were performed on a much less powerful machine (Intel Centrino 1.6GHZ with 768M RAM).

The sensitivity of the UMLDiff and ETGM algorithms is linked to the number of parameters and possible settings. In UMLDiff, there are two thresholds that determine its behavior. As our algorithm, UMLDiff proposes a setting that produced good results on JFreeChart. Our setting was tested with good results on three different systems of different sizes and domains.

Finally, unlike UMLDiff, we do not support the detection of refactoring actions like moves of methods and attributes among classes. However, while UMLDiff relies heavily on class and package names for its initialisation, we also exploit relations. A more thorough comparison of the two algorithms would require extensive experimentation and validation and will be subject to future work.

\section{RELATED WORK}

There exist several pieces of work in the literature related to the analysis of software evolution $[1,8,15,18,22,23,25,26,27]$. In general, differencing algorithms compute the delta between two releases of a system using a flat representation (i.e., considering a system as a sequence of lines of code) or using various underlying representations (e.g., logic facts).

\subsection{Differencing Algorithms at File Level}

Several algorithms have been presented in the literature (e.g., [11]) that model software evolution at the level of lines of code and that report added and deleted lines. These algorithms are relatively simple to implement (e.g., using the Unix diff algorithm) and to apply. Yet, as noted in $[8,27]$, they are not adapted to study the evolution of a system. Indeed, when used in the context of software evolution, these algorithms miss important information. For example, when a class is renamed, the Unix diff algorithm would report the change as the original class being deleted and a new class being added, while a developer would be interested to understand the renaming [27].

Canfora et al. [8] presented a novel line-differencing algorithm, ldiff, that overcomes Unix diff limitations to identify changed text lines. ldiff is a language-independent algorithm that can be used for tracking the evolution of classes as a sequence of lines to track the evolution of source clones or to monitor vulnerable instructions of networking systems. It could also be used to analyze different artifacts, such as source code, use cases, and test cases. ldiff focuses on tracking blocks of lines across file releases, trying to distinguish between line changes and additions/deletions. Our ETGM algorithm works at a higher level of abstraction, identifying the addition, removal, and change in classes, methods, attributes, and their relations. 
Godfrey et al. $[15,25]$ proposed an algorithm, Beagle, to analyze the evolution of software systems. Their algorithm works at the file-structure level, using origin analysis [25], while our algorithm considers the design of the target system releases and compares graphs. Origin analysis is a process that borrows techniques from software clone detection and tries to decide if a class is introduced in a new release or if it should be seen as the same class that has changed during the evolution from the old release to the new one.

\subsection{Differencing Algorithms at the Design Level}

The problem of detecting changes between the designs of subsequent releases of systems has been already studied in the past by Antoniol et al. [1]. As in our proposal, their algorithm recovers the design from the source code in an intermediate representation and compares it with subsequent releases. Differently from us, it uses a maximum match algorithm [9] applied to a bipartite graph. Nodes in the bipartite graph are the classes of the two releases and the similarity between them is derived from class and attribute/method names by means of string edit distance. The algorithm did not deal with the class relations.

Xing and Stroulia $[26,27]$ proposed UMLDiff for matching different versions of a system and which has been implemented as an Eclipse plug-in. As mentioned in the previous section, there are some commonalities between the two algorithms. We believe that our algorithm is more general because it works on graphs. It can be easily extended to other software artifacts that can be represented as graphs.

In [20], we proposed and applied for the first time a Google-inspired ETGM algorithm [19] to analyze software evolution. Many features of the algorithm presented in this paper were already present in our former tabu implementation [20,21]. The algorithms use the same neighborhood function and the same tabu search. Both algorithms combine the objective function with extra information related to structural similarity between pairs of vertices. However, there are three major differences between the two contributions.

The first difference is in the definition of the similarity measures used to guide the search. In the former algorithm, two different kinds of similarity measures were combined: a local measure and a structural similarity based on PageRank ${ }^{11}$ [4]. In our novel algorithm, we use a single measure of local similarity. As it now takes edge labels into account, this measure is more precise and we avoid the use of PageRank. Therefore, we simplify the definition of similarity without loss of accuracy.

The second difference concerns the use of the similarity measures. In the former algorithm, the search is initialized using an empty solution; then, the similarity measures are combined with the objective function throughout the search using a weighting parameter [20,21]. In the novel algorithm, there are now two successive phases: the initialization phase and the tabu search. Structural information are used only during initialization; then, only the objective function is used in the tabu search. As a result, a weighting parameter is no longer necessary. In

\footnotetext{
${ }^{11}$ PageRank is one of the main components behind the first versions of Google, it basically measures the relative importance of each element of a hyperlinked set and assigns it a numerical weighting.
} 
addition, initialization prevents the novel algorithm from proceeding through a time-consuming step-by-step building of a solution, as shown empirically in Section 3.

The third difference is in the empirical evaluation. In [21], we demonstrated the applicability of the ETGM algorithms for studying software evolution using 144 versions corresponding to six years of Mozilla evolution. This paper extends this previous work by applying the novel algorithm to five systems to answer research questions dealing with the algorithm sensitivity to parameters, its scalability, and its stability. Sensitivity of the ETGM algorithm as well as its scalability were not treated in our previous work.

\section{3. $\quad$ Logic-based Representations}

Some algorithms exist that use logic-based facts to analyze the evolution of software systems. For example, LSdiff [18] groups the results representing the differences and infers logic rules to discover and represent systematic structural changes.

A fact extractor (as for example grok [17]) is first applied on two releases of a same system. Then, LSdiff computes the differences between extracted facts to obtain fact-level differences. These differences are then condensed into simpler rules. The purpose of LSdiff is similar to ours but (1) the underlying representations are different (logic facts vs. graphs), (2) the used algorithms are different, and (3) the results are shown at two different levels of abstraction (logic rules grouping similar code changes vs. list of additions, removals, and changes).

\section{CONCLUSION}

Analyzing and understanding the evolution of large object-oriented software systems is an important task for developers. This task was addressed in previous work using custom algorithms. In this paper, we proposed to use a general-purpose error-tolerant graph matching (ETGM) algorithm to analyze software evolution. We implemented a novel ETGM algorithm, which can identify in large systems the classes that remain stable across releases and those that change, with respect to their attributes, methods, and relations with other classes.

First, we described the theory and implementation of our novel ETGM algorithm. We detailed the process of building an oracle to validate matching results and applied this process on three systems, Rhino, ArgoUML, and Azureus. Finally, we introduced three research questions and carried out empirical studies to answer them.

During the study, we applied our novel algorithm on five systems: Rhino, ArgoUML, Azureus, the Mozilla Web browser, and Eclipse. First, we studied the impact of the parameter values on the precision and recall of our algorithm. Then, using selected parameter values, we studied the performance and stability of our algorithm. On all systems but Eclipse, our algorithm computes a single solution in less than 30 seconds and is, thus, substantially faster than our previous algorithm [21]. The improvement in terms of performance led to a negligible decrease of the stability of the solutions. Thus, we showed that the algorithm presented in this paper outperforms our previous work and is able to fulfill the task of identifying (un)stable classes in large object-oriented software systems. 
Future work will be devoted to extend our algorithm to handle many-to-one, one-to-many, and many-to-many class evolution patterns. We also plan to extend and evaluate our algorithm on other artifacts, such as activity and state diagrams.

\section{Acknowledgment}

This research was partially supported by the Natural Sciences and Engineering Research Council of Canada through the Research Chairs in Software Evolution and in Software Patterns and Patterns of Software.

\section{REFERENCES}

1. G. Antoniol, G. Canfora, G. Casazza, and A. De Lucia. Maintaining traceability links during objectoriented software evolution. Software - Practice and Experience, 31:331-355, April 2001.

2. G. Antoniol, R. Fiutem, and L. Cristoforetti. Design pattern recovery in object-oriented software. Sixth Workshop on Program Comprehension, pages 153-160, June 1998.

3. G. Antoniol, Y.-G. Gueheneuc, E. Merlo, and P. Tonella. Mining the lexicon used by programmers during sofware evolution. In IEEE International Conference on Software Maintenance (ICSM 2007), pages 14-23, Oct. 2007.

4. Sergey Brin and Lawrence Page. The anatomy of a large-scale hypertextual web search engine. Comput. Netw. ISDN Syst., 30(1-7):107-117, 1998.

5. M. Bunge. Treatise on Basic Philosophy: Vol. 3: Onthology I: The Furniture of the World. Reidel, Boston MA, 1977.

6. M. Bunge. Treatise on Basic Philosophy: Vol. 4: Onthology II: A World of Systems. Reidel, Boston MA, 1979.

7. H. Bunke. On a relation between graph edit distance and maximum common subgraph. Pattern Recogn. Lett., 18(9):689-694, 1997.

8. G. Canfora, L. Cerulo, and M. Di Penta. Tracking your changes: A language-independent approach. IEEE Software, 26(1):50-57, 2009.

9. T. H. Cormen, C. E. Leiserson, and R. L. Rivest. Introductions to Algorithms. MIT Press, 1990.

10. ECMA. ECMAScript Standard - ECMA-262 v3. ISO/IEC 16262, 2007.

11. S.G. Eick, J.L. Steffen, and E.E Summer. SEESOFT - a tool for visualizing line-oriented software statistics. IEEE Transactions of Software Engineering, 18:957-968, 1992.

12. W. B. Frakes and R. Baeza-Yates. Information Retrieval: Data Structures and Algorithms. Prentice-Hall, Englewood Cliffs, NJ, 1992.

13. F. Glover. Tabu search-part I. ORSA Journal on Computing, 1 (3):190 - 206, 1989.

14. M. Godfrey and Q. Tu. Tracking structural evolution using origin analysis. In Proc. of the International Workshop on Principles of Software Evolution, pages 117-119. IEEE Computer Society, 2002.

15. M.W. Godfrey and L. Zou. Using origin analysis to detect merging and splitting of source code entities. IEEE Transactions on Software Engineering, 31(2):161-181, Feb. 2005.

16. Y.G. Guéhéneuc and G. Antoniol. Demima: A multi-layered framework for design pattern identification. Transactions on Software Engineering, 34(5):667-684, December 2008.

17. R.C. Holt. Structural manipulations of software architecture using tarski relation algebra. In Proc. of the Working Conference on Reverse Engineering, pages 210-219. IEEE Computer Society, 1998.

18. M. Kim and D. Notkin. Discovering and representing systematic code changes. In Proceedings of the International Conference on Software Engineering, pages 309-319, 2009.

19. S. Kpodjedo, P. Galinier, and G. Antoniol. A Google-inspired error correcting graph matching algorithm. Technical Report EPM-RT-2008-06, Ecole Polytechnique de Montreal, 062008.

20. S. Kpodjedo, F. Ricca, P. Galinier, and G. Antoniol. Error correcting graph matching application to software evolution. In Proc. of the Working Conference on Reverse Engineering, pages 289-293. IEEE Computer Society, 2008. 
21. S. Kpodjedo, F. Ricca, P. Galinier, and G. Antoniol. Recovering the evolution stable part using an ECGM algorithm: Is there a tunnel in mozilla? In Proceedings of European Conference on Software Maintenance and Reengineering, pages 179-188, Los Alamitos, CA, USA, 2009. IEEE Computer Society.

22. M. Lanza, H. Gall, and P. Dugerdil. Evospaces: Multi-dimensional navigation spaces for software evolution. In Proc. of the Conference on Software Maintenance and Reengineering, pages 293-296, 2009.

23. D. Mandelin, D. Kimelman, and D.M. Yellin. A bayesian approach to diagram matching with application to architectural models. In Proceedings of the International Conference on Software Engineering, pages $222-231,2006$.

24. W. Tsai and K. S. Fu. Error-correcting isomorphism of attributed relational graphs for pattern analysis. IEEE Trans. on Systems, Man, and Cybernetics, 9:757 - 768, 1979

25. Q. Tu and M.W. Godfrey. An integrated approach for studying architectural evolution. In Proceedings of the 10th International Workshop on Program Comprehension (IWPC), pages 127-136. IEEE Computer Society, 2002.

26. Z. Xing and E. Stroulia. Analyzing the evolutionary history of the logical design of object-oriented software. IEEE Transactions on Software Engineering, 31(10):850-868, October 2005.

27. Z. Xing and E. Stroulia. UMLDiff: an algorithm for object-oriented design differencing. In $A S E$ '05: Proceedings of the 20th IEEE/ACM international Conference on Automated software engineering, pages 54-65, New York, NY, USA, 2005. ACM.

Segla Kpodjedo received his Engineer Diploma in computer engineering

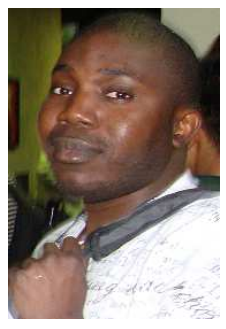
in 2005 from UTBM, France. From 2006 to 2007, he pursued and completed his Master of Science Applied (M.Sc.A) at LARIM, Ecole Polytechnique de Montreal, Canada. Since 2007, he is a $\mathrm{Ph}$. D student working under the supervision of Professors Philippe Galinier and Giulio Antoniol at SOCCERLab, Ecole Polytechnique de Montreal, Canada. His current work mainly revolves around search based software engineering. His research interests include software evolution, fault prediction in software systems, approximate graph matching, resolution of NP-hard problems using metaheuristics, multi-agent systems and mobile networks.

Filippo Ricca collaborates with the Software Engineering Group of DISI

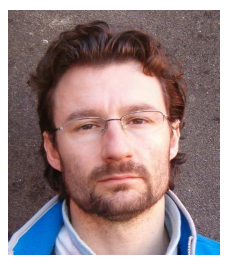
(Department of Computer and Information Science) at the University of Genova, Italy. He received his laurea degree in Computer Science from the University of Genova, Italy, in 1994, and his $\mathrm{PhD}$ degree in Software Engineering from the same University, in 2003, with the thesis "Analysis, Testing and Re-structuring of Web Applications". From 1999 to 2006, he worked with the Software Engineering group at ITC-irst (now FBK-irst), Trento, Italy. During this time he was part of the team that worked on Reverse engineering, Re-engineering and Testing. His current research interests include Reverse engineering, Empirical studies, Web applications and Testing. The reseach is mainly conducted through empirical methods such as case studies, controlled experiments and surveys.

Philippe Galinier has studied computer science at Montpellier II

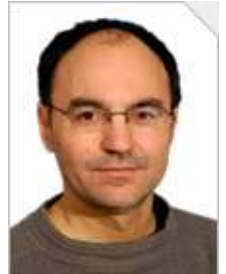
University (France) and received his Ph.D. in 1999. He is now associate professor in the department of Computer and Software Engineering at Ecole Polytechnique of Montreal (Canada). His interest deals with the solution of large-scale difficult (NP-hard) combinatorial problems by using metaheuristics, such as neighborhood search (notably tabu search) and evolutionary algorithms (notably memetic algorithms). The targeted problems include both academic problems (notably graph-related problems 
such as graph coloring) and real-world applications (notably in the areas of telecommunication networks and software engineering).

Giuliano Antoniol (Giulio) received his Laurea degree in electronic

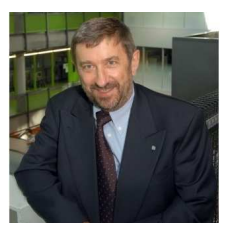
engineering from the Universita' di Padova, Italy, in 1982. In 2004 he received his $\mathrm{PhD}$ in Electrical Engineering at the Ecole Polytechnique de Montreal. He worked in companies, research institutions and universities. In 2005, he was awarded the Canada Research Chair Tier I in Software Change and Evolution.

He has participated in the program and organization committees of numerous IEEE-sponsored international conferences. He served as program chair, industrial chair, tutorial, and general chair of international conferences and workshops. $\mathrm{He}$ is a member of the editorial boards of four journals: the Journal of Software Testing Verification \& Reliability, the Journal of Information and Software Technology, the Journal of Empirical Software Engineering and the Software Quality Journal.

Dr Giuliano Antoniol served as Deputy Chair of the Steering Committee for the IEEE International Conference on Software Maintenance. He contributed to the program committees of more than 30 IEEE and ACM conferences and workshops, and he acts as referee for all major software engineering journals.

He is currently Full Professor at the Ecole Polytechnique de Montreal, where he works in the area of software evolution, software traceability, search based software engineering, software testing and software maintenance.

Yann-Gael Guéhéneuc is associate professor at the Department of

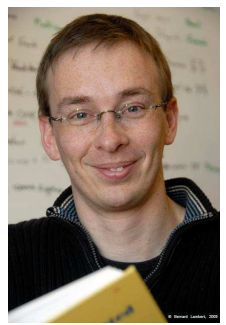
computing and software engineering of Ecole Polytechnique of Montreal where he leads the Ptidej team on evaluating and enhancing the quality of object-oriented programs by promoting the use of patterns, at the language-, design-, or architectural-levels. In 2009, he was awarded the NSERC Research Chair Tier II on Software Patterns and Patterns of Software. He holds a Ph.D. in software engineering from University of Nantes, France (under Professor Pierre Cointe's supervision) since 2003 and an Engineering Diploma from cole des Mines of Nantes since 1998. His Ph.D. thesis was funded by Object Technology International, Inc. (now IBM OTI Labs.), where he worked in 1999 and 2000. His research interests are program understanding and program quality during development and maintenance, in particular through the use and the identification of recurring patterns. He was the first to use explanation-based constraint programming in the context of software engineering to identify occurrences of patterns. He is interested also in empirical software engineering; he uses eye-trackers to understand and to develop theories about program comprehension. He has published many papers in international conferences and journals. He is IEEE Senior Member since 2010. 